\title{
PERSPECTIVE CORRECTION FOR IMAGE PROCESSING
}

\author{
Romana OANCEA*, Vlad BÂRSAN**, Cristian MOLDER*** \\ *"Nicolae Bălcescu" Land Forces Academy, Sibiu, Romania, \\ **"Lucian Blaga" University of Sibiu, Sibiu, Romania \\ ****Military Technical Academy, Bucharest \\ oancea.romana@gmail.com
}

\begin{abstract}
The paper proposes an algorithm for applying the perspective correction of images containing human faces. Based on the coordinates of the two eyes, of the center of the mouth and of a point on the line of the mouth, one can estimate the angle of inclination of the face. After applying the perspective correction, the area corresponding to the face can be used by a face recognition algorithm based on aspect in order to improve the good recognition rate.
\end{abstract}

\section{Key words: eye detection, mouth detection, perspective deformation, perspective transformation.}

\section{Introduction}

Currently, facial recognition algorithms from intensity images can be classified into two categories [1-3]: (1) the holistic model or photometric recognition and (2) featurebased or geometric recognition.

Face recognition algorithms based on features are founded on the construction of a human face model, able to capture the changes of the faces. The a priori knowledge about the human face is used to build models. For example, the modelbased matching starts from the calculation of the relative distances and positions of distinctive facial features like the eyes, the mouth, the nose etc. The distances can be calculated using different metrics. The recognition algorithms based on a model usually contain three stages: the construction of the model, the fitting of the model on a given face and the use of the model parameters as a vector of features for determining the similarity between the questioned face and the prototype faces from the database for recognition.
The holistic approaches detect a face using the global features of the face and not the local characteristics. These techniques are also divided into two categories: statistical and AI approaches [3].

The most known statistical holistic algorithms - Principal Component Analysis (PCA) [4] and its variant algorithms [22], Fisher's Linear Discriminant Analysis (LDA) [5-6], and the Independent Component Analysis (ICA) [7] use the entire face area in the recognition process. Starting from the face area one constructs the image vector which is projected on the basic vectors that are built based on prototype faces. By the projection of the image vector based on basic vectors, the resulting coefficients are used in the form of a representation of the image through a set of characteristics. The matching score between a test image and a prototype image is determined by means of the coefficients of the projections; the higher the score, the better the matching degree. 
The main advantage of this method is that it focuses not only on one area or on certain points of interest [8], but the results are directly influenced by scale, illumination and pose [9].

Most public databases containing faces are background controlled. This paper proposes an algorithm to detect faces and then to normalize them in an uncontrolled context, so that the determined area can be used by an algorithm for recognition so as to lead to an increased good recognition rate. In order to assess the results, a database consisting 1,068 Internet images representing a single person was created. The individuals can be photographed in one or more situations, and for each image the maps corresponding to the eyes, respectively the mouth, were generated.

\section{Face detection based on facial features}

The detection of the face in color images, with uncontrolled context, was based on the eyes and mouth facial features. For eye detection, the color maps [10], the symmetry map [11] and the image intensity are combined into a single selection criterion [12]:

$$
I_{E}(x, y)=E(x, y)^{k_{E}}+[1-S(x, y)]^{k_{S}}+[1-I(x, y)]^{k_{I}}
$$

where $k_{E}, k_{S}, k_{I}$ are the weights associated to the detection map of the eyes $E(x, y)$, to the symmetry map $S(x, y)$ and to the intensity of the image $I(x, y) ; k_{E}, k_{S}, k_{I}$ are improper fractions.

The area corresponding to a potential eye in the analyzed image will have a higher value on the color map $E(x, y)$ and lower values on the symmetry maps $S(x, y)$ and on intensity maps $I(x, y)$, respectively. A maximum value of the $I_{E}$ criterion corresponds to a maximum of probability for locating an eye on the $(x, y)$ coordinate. The pixel candidates as potential eyes in the image represent local minimum values and are determined by means of the Watershed transform based on which one can determine a single local minimum value at region level. Thus, first we determine the area containing local minimum values and retain only the first two values.

The major problems that occurred in the detection of the eyes, based on color maps, symmetry and intensity, refer to the image resolution [9] and to points of local extrema detected in the corners of the image.

In order to eliminate the errors generated by the resolution, various heights of the image were taken into consideration (in the images that contain faces, the ratio width/height $<1$ ), and the best results were obtained if the height was between 325 pixels and 350 pixels $(80.24 \%$ and $80.43 \%$ good detection rate, respectively) due to the symmetry elements that directly affect the image resolution. Taking into account a height $\mathrm{H}=325$ pixels resolution, the images that have a lower height will remain unchanged, and those with a higher height will be rescaled by bilinear interpolation at a value $\mathrm{H}$ [23].

With regard to the symmetry elements detected at the extremities of the images, they were eliminated during a preprocessing procedure applied to image in order to isolate the skin area. Initially, from the color image in the HSV space, we determined the skin area by using the Bayesian approach with the MAP (maximum aposteori) technique [13-16].

The determination of the candidate area for detecting and locating the mouth is based on the fact that this type of feature has a strong red component as well as a small amount of blue in comparison to the other facial regions. Therefore, in the mouth region, the chrominance component $\mathrm{Cr}$ is higher than the $C b$ component and has a reduced response of the $\mathrm{Cr} / \mathrm{Cb}$ parameter and a high response for $\mathrm{Cr}^{2}$. One can build the map associated to the candidate area for the mouth M [10]

$$
\begin{aligned}
& M=C_{r}^{2} \cdot\left(C_{r}^{2}-\eta \cdot \frac{C_{r}}{C_{b}}\right)^{2} \\
& \eta=0.95 \cdot \frac{\frac{1}{n} \sum_{(x, y) \in F} C_{r}(x, y)}{\frac{1}{n} \sum_{(x, y) \in F} C_{r}(x, y) / C_{b}(x, y)}
\end{aligned}
$$

where $\mathrm{Cr}^{2}$ and $\mathrm{Cr} / \mathrm{Cb}$ are normalized in the field $[0 ; 255], n$ is the number of pixels in the face mask $F$, and $\eta$ is estimated as the 
ratio between the average values of the $\mathrm{Cr}^{2}$ and $\mathrm{Cr} / \mathrm{Cb}$ components.

Unlike the eye, which represents a great variation in color and shape, the mouth has a relatively uniform color and shape, regardless of the facial expression and illumination. The shape of the mouth is elongated and thin and cannot be determined by symmetry elements.

After applying the maps corresponding to the two eyes, and to the center of the mouth, respectively, one can obtain detection errors both at the level of the eyes and the level of the mouth (Fig. 1). For the database under consideration, the good detection rate of the mouth, with the mask described in relation 2 and a scaling of the image to value $\mathrm{H}=325$ pixels, reached values of over $95.88 \%$; the errors occurred only in the case of red artefacts in the skin area.

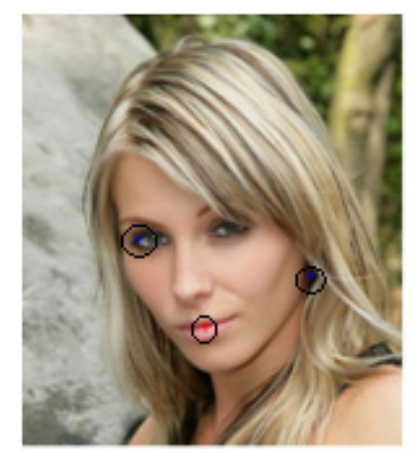

Figure 1. The detection of eyes (a correct eye and an erroneously detected eye) and of the center of the mouth based on the associated maps

The next step is to reduce the number of omissions in the detection of the eyes, considering that the mouth is large enough to ensure at least its position information. Based on the anthropometric measurements of the distance between the eyes and mouth and the middle of the line of the eyes [1718], one has to check if the detected features may form a triangle of the face (relation 4)

$$
\begin{aligned}
& 0.8<\frac{D m}{D e}<1.6 \\
& 35^{\circ}<\alpha_{M E}<65^{\circ}
\end{aligned}
$$

where $D e$ is the line determined by the two eyes, $D m$ is the segment determined by the center of the mouth and the middle of the segment joining the two eyes, $\alpha_{M E}$, the angle determined by the two segments joining the center of the mouth with the left eye, and the right eye, respectively.

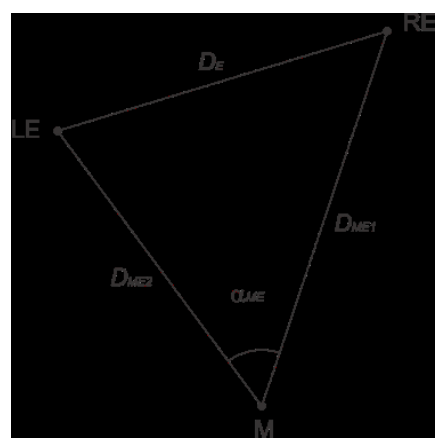

Figure 2. Triangle of the face determined by the center of the mouth and the two eyes

The three determined points will be validated only if they cumulatively meet the conditions described in relation 4 . If the conditions are not validated, it is necessary to identify the correct eye first, and then the position of the undetected eye will be estimated starting from the positions of the mouth and that of the correct eye. If the conditions are not validated, it is necessary to identify the correct eye first and then one can estimate the position of the undetected eye starting from the positions of the mouth and of the correct eye.

For the database under consideration, under the conditions of rescaling the images at a height $\mathrm{H}=325$ pixels and using the masks constructed a priori for the eyes and mouth, a correct detection rate of the three facial features $\mathrm{TDR}=71.63 \%$ was obtained, a rate that was improved by identifying the correct eye and by estimating the position of the second eye.

The criterion of validation/selection of the correct eye is based on the evidence that the eye has a white component and has an elliptical shape, thus the white coefficient is calculated based on the radius disks De/4 determined by the coordinates of the eyes and the correct eye is considered the area containing the greatest amount of white 


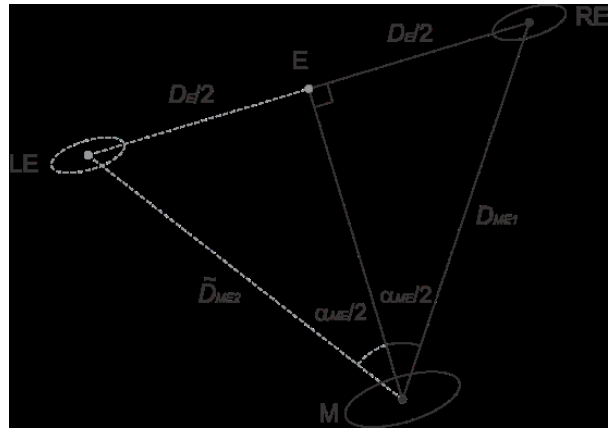

Figure 3. Estimating the position of the undetected angle

Considering that the position of the mouth and that of an eye are correct, one can estimate both the position of the undetected eye and the rotation angle of the face in the image, if it does exist. The proposed algorithm to estimate the position is the following (Figure 3):

- the length of the segment is calculated, $D_{M E 1}$ or $D_{M E 2}$ as appropriate;

- the midpoint $E$ of the segment $D_{E}$ considering $\alpha_{M E} / 2=45^{\circ} / 2$ and the ratio $D_{M} / D_{E}=1.25$ are calculated;

- the length of the segment $D_{E}$ is calculated as twice the length of the segment between point $\mathrm{E}$ and the position of the correctly detected eye:

$D_{E}=2 D_{M E} \times \sin \left(\alpha_{M E} / 2\right)$

- the position of the undetected eye is determined starting from the segment $D_{E}$, by rotation with angle $\beta$ and the translation corresponding to the triangle of the face where appropriate

$$
\beta=a \tan \left(\frac{y_{D_{M}}-y_{D_{E}}}{x_{D_{M}}-x_{D_{E}}}\right)
$$

The results obtained after estimating the undetected eye are good and the TDR has reached a value of $84.46 \%$.

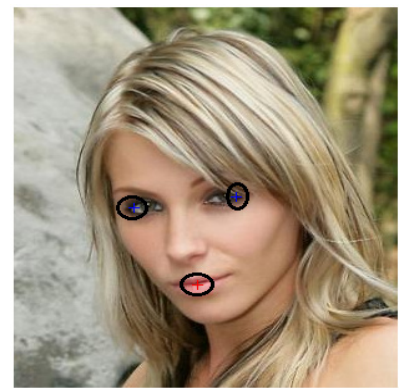

In brief, the algorithm for locating the facial features based on the fact that the features are arranged in the form of a triangular pattern [19-21], can be summarized as follows:

- detecting the skin area in color images using a Bayesian classification by means of the MAP method; If the limits of the skin area are adjacent to the edges of the image, we will board it with 10 pixels up to the edges of the image. The facial features will be detected only in the delineated skin area.

- locating the eyes - using the map of the chrominance, the symmetry map and the image intensity (rel. 1);

- on the basis of the map corresponding to the mouth (rel. 2), the center of the mouth is determined;

- checking the triangle of the face - the coordinates of the eyes and the coordinate of the mouth form a facial-feature-triangle (approximately an isosceles triangle - for a face oriented forward); a distorted triangle for a face that does not face forward).

- if the three coordinates (eyes, mouth) do not cumulatively meet the conditions in relation 4 , the correct eye is validated based on the histogram of the white component, the eye with the greatest amount of white is retained - and the position of the undetected eye is estimated.

- if the three coordinates cumulatively meet the conditions of relation 4 , one can estimate the angle of the face for normalization.

\section{Estimating the angle of inclination of the face}

The concrete estimation of the angle of inclination can be done by selecting an area in the image corresponding to the mouth, starting from the detected point of the mouth, so that for determining the angle, one should apply the Radon transform. The Radon transform [25] (rel. 7) applied to a binary image can determine the angle of inclination of all the existing lines. After applying the transform, a line will be characterized by two parameters: $\theta$ - the angle of inclination and $\rho$ - the minimum 
distance from the origin of the system of coordinates.

$R(\rho, \theta)=\int_{-\infty}^{\infty} f(\rho \cdot \cos \theta-s \cdot \sin \theta, \rho \cdot \sin \theta+s \cdot \cos \theta) d s(7)$

The application of the Radon transform to a binary image of size 250x259 (Fig. 5) enables the determination of the angle of inclination, an angle determined as the maximum of the projections of the intensities.
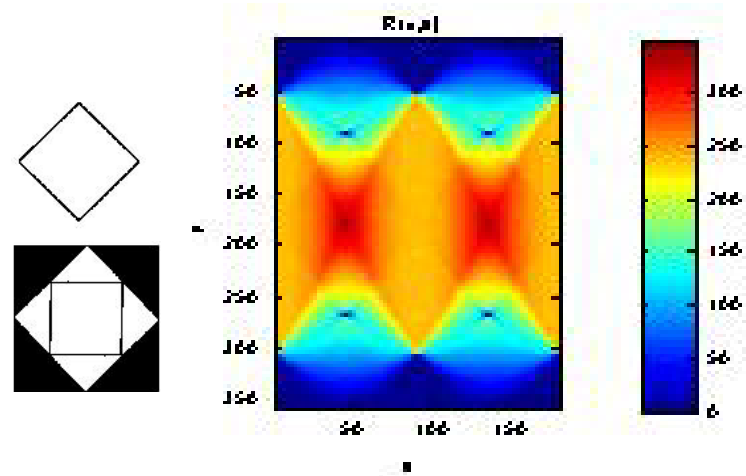

Figure 5 The Radon transform applied to a lozenge and the determination of the angle of inclination

The Radon transform applied to an image ensures the connection between the rectangular coordinates $(x, y)$ and the space of projections $(\rho, \theta)$. The angle of inclination of the image, if it does exists, corresponds to the projection of the maximum variation [24].

Thus, the Radon transform will be applied to a binary image representing the mouth contour detection in the grayscale version of the corresponding color region (Fig. 6). Since the size of the face and therefore of the mouth are not known at this time, one can choose a window to cut in the region around the detected point of the mouth with the following dimensions:

$$
\begin{aligned}
& W_{w}=\left[\frac{W}{6}\right]+1 \\
& H_{w}=3 W / 4
\end{aligned}
$$

where $W_{w}$ and $H_{w}$ are odd imposed dimensions of the window to be cut, and $\mathrm{W}$ is the width of the image (assuming the most usual case where the mouth is disposed horizontally).

The contour detection will be done after a filtering with a Gaussian low-pass filter to remove the disturbing contour elements, like the small grooves specific to the skin.

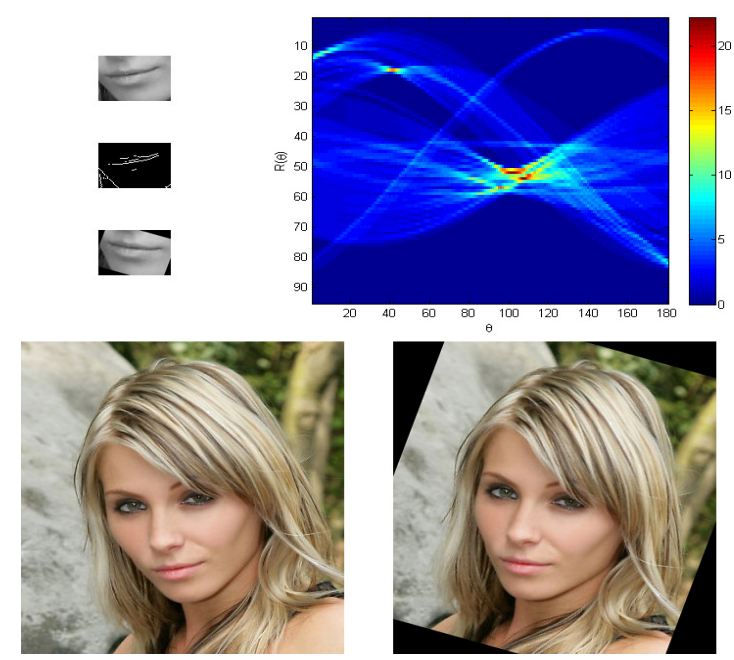

Figure 6. Example of determining the rotation angle of the face by means of the Radon transform and of the contour of the detected mout 
The selection of the height of the region corresponding to the mouth will be $2 / 3$ above the detected point and $1 / 3$ below it (Fig. 7). This is necessary because in most cases, the detected point is on the lower lip. Moreover, in the case of the facial expressions, the upper lip changes its horizontal form less, enabling a more accurate detection of the angle of orientation.

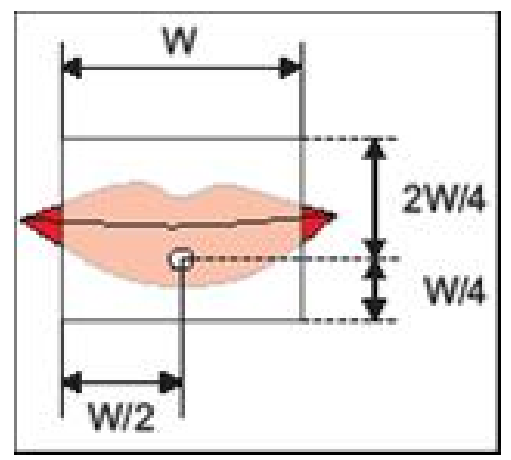

Figure 7. The selection of the calculation region of the Radon transform for the detection of the inclination angle of the mouth

The images can contain different obstructions caused, for example, by strands of hair or other objects, so that it is more practical to choose the detection of the mouth contour in a color space which illustrates its prominence. Therefore, the conversion of the analyzed image in the $C b^{2} / Y$ plan of the $Y C b C r$ space is preferred.

Moreover, the mask is filtered through a $G(x, y)$ low-pass Gaussian filter with the size $W / 8 \times W / 8$, and uniform dispersion, and the $Y$ and $C b$ plans are equalized in contrast by means of the equalization operation of the $H E[\cdot]$ histogram.

$M_{C}=G\left(\frac{H E\left[C b^{2}\right]}{H E[Y]}\right)$

where:

- $H E\left[C b^{2}\right]$ - histogram equalization for the chrominance component $C b^{2}$,

- HE[Y] - histogram equalization for luminance component $Y$;

- G(.) - low-pass Gaussian filter

Applying this technique in order to detect the rotation angle based on the orientation of the lips has the advantage that it can be used during any stage of processing, the only condition being that of accurate detection of the mouth point. The method is useful both in the context of clear images and in the case of those that have obstructions or distortions caused by different facial expressions.

Various problems can occur in the situations when it is not facing front, when the triangle of the face undergoes a deformation caused by perspective. Thus, although the eye line is parallel to that of the mouth, they become convergent in the projected image. The application of the rotation with the Radon transform will determine the achievement of a correct orientation of the mouth, while the eye line will continue to have an inclination angle due to perspective. The elimination of this drawback can be done only in the situations when we know the position of the mouth and of the eyes by applying a warping bilinear interpolation correction (Fig. 8).

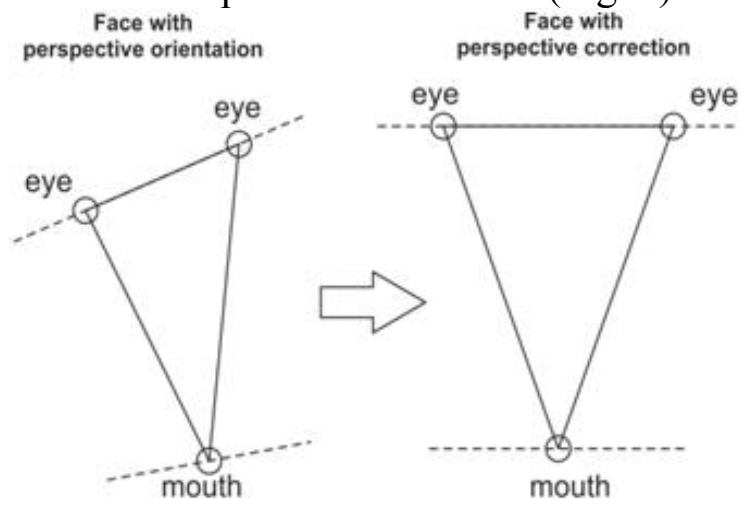

Figure 8. The correction of the perspective deformation by warping by means of the detected points of the triangle of the face

\section{The perspective correction}

The perspective deformations occur in situations when the subject is located at a certain angle in relation to the objective of the camera. As a result, the objects closer to the camera are larger than those that are farther. The perspective deformation can be corrected using an inverse perspective transformation. This type of transformation carries out the association (the mapping) between two quadrilaterals, one in the distorted image and one in the corrected 
image (Fig. 9). Unlike other situations where the parallelism of the straight lines was required, it is not necessary in this case.

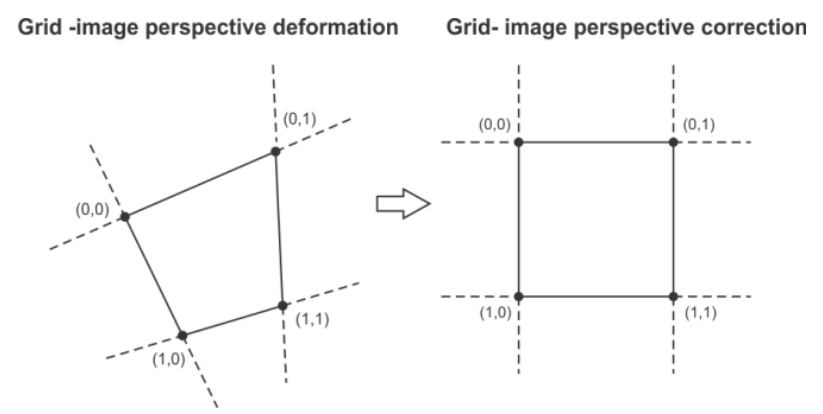

Figure 9. Rectangular grid for applying the perspective deformation correction

$\left[\begin{array}{l}x^{\prime} \\ y^{\prime} \\ w^{\prime}\end{array}\right]=\left[\begin{array}{lll}m_{11} & m_{12} & m_{13} \\ m_{21} & m_{22} & m_{23} \\ m_{31} & m_{32} & m_{33}\end{array}\right] \times\left[\begin{array}{l}x \\ y \\ 1\end{array}\right]$

The perspective transform is defined by a transformation matrix of $3 \times 3$ size which enables the projection of the homogenous coordinates in the distorted image, $\left(\begin{array}{lll}x & y & 1\end{array}\right)$, in the new coordinates of the corrected image, $\left(x^{\prime} y^{\prime} w^{\prime}\right)$. In order to transform the new coordinates into non- homogeneous coordinates, the $x^{\prime}$ and $y^{\prime}$ coordinates are divided to the $w^{\prime}$ coefficient.

$$
\begin{aligned}
x^{\prime} & =\frac{m_{11} x+m_{12} y+m_{13}}{m_{31} x+m_{32} y+m_{33}} \\
y^{\prime} & =\frac{m_{21} x+m_{22} y+m_{23}}{m_{31} x+m_{32} y+m_{33}}
\end{aligned}
$$

In order to construct the correction grid at least 4 points forming the association quadrilateral are required. In the present case, however, only the positions of the three points that make up the triangle of the face are known. It is necessary, therefore, to make an algorithm that enables the estimation of two corresponding points starting from the point of detection of the mouth, while the other two points need to be considered eye detection points. It is also possible to determine the angles of the segment that connects the eyes and the mouth segment by calculation.

From the anthropometric analysis of the face, the following formal relations between the facial elements are known or at least can be empirically estimated:
- the mouth length is $2 / 3$ of the distance between the extremities of the two eyes;

- the ratio between the distance of the eyes and the distance from the mouth to the middle of the distance of the eyes is 1.25 (average determined value);

- the vertical angle of the segment connecting one eye and the mouth is $22.5^{\circ}$ (average value determined experimentally). It is necessary to estimate the length of the mouth in order to determine the 4 points necessary to the correction grid (Fig. 10). If it is assumed that the point of detection of the mouth is at the middle of the segment of the mouth, the length of the mouth is estimated as $2 / 3$ of the segment connecting the coordinates of the two eyes $E_{l}$ and $E_{2}$.

\section{Perspective deformation Perspective correction}

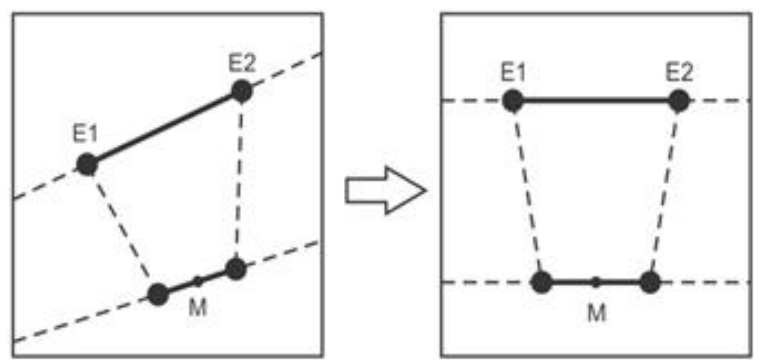

Figure 10. Rectangular grid for applying the perspective deformation correction

In order to implement the warping, a quadruple parameterization is carried out starting from the four corresponding points on the grid. Four line segments parametric to the portions are created and are defined according to formula (14):

$$
\begin{aligned}
& c_{1}(u)=E_{1}+u \cdot\left(E_{2}-E_{1}\right) \\
& c_{2}(v)=M_{1}+v \cdot\left(M_{2}-M_{1}\right) \\
& c_{3}(w)=E_{1}+w \cdot\left(M_{1}-E_{1}\right) \\
& c_{4}(k)=E_{2}+k \cdot\left(M_{2}-E_{2}\right)
\end{aligned}
$$

where $E_{1}, E_{2}, M_{1}$ and $M_{2}$ are the coordinates of the points of the eyes and mouth from the initial image, and $u, v, w$ and $k$ are the parameters associated to the curves, defined according to relation (15): 
$u=\frac{y-y_{E_{1}^{\prime}}}{y_{E_{2}^{\prime}}-y_{E_{1}^{\prime}}}$

$v=\frac{y-y_{M_{1}^{\prime}}}{y_{M_{2}^{\prime}}-y_{M_{1}^{\prime}}}$

$w=\frac{x-x_{E_{1}^{\prime}}}{x_{M_{1}^{\prime}}-x_{E_{1}^{\prime}}}$

$k=\frac{x-x_{E_{2}^{\prime}}}{x_{M_{2}^{\prime}}-x_{E_{2}^{\prime}}}$

where $E_{1}^{\prime}, E_{2}^{\prime}, M_{1}^{\prime}$ and $M_{2}^{\prime}$ are the coordinates of the points of the eyes and mouth from the final image.

We determined the intermediary points $\left(x_{i}\right.$; $\left.y_{i}\right), i=1 . .4$, on the four curves, $c_{i}, i=1 . .4$, and by means of these we determined the equations of two straight lines whose parameters are defined according to equations (16):

$$
\begin{aligned}
& b_{1}(u, v)=\frac{y_{1}(u) \cdot x_{2}(v)-x_{1}(u) \cdot y_{2}(v)}{x_{2}(v)-x_{1}(u)} \\
& a_{1}(u, v)=\frac{y_{1}(u)-b_{1}(u, v)}{x_{1}(u)} \\
& b_{2}(w, k)=\frac{y_{3}(w) \cdot x_{4}(k)-x_{3}(w) \cdot y_{4}(k)}{x_{4}(k)-x_{3}(w)} \\
& a_{2}(w, k)=\frac{y_{3}(w)-b_{2}(w, k)}{x_{3}(w)}
\end{aligned}
$$

The current coordination point (Fig. 10) is given by the intersection of the two segments defined in relation (17) as solution of the system:

$$
\begin{aligned}
& y(u, v)=a_{1} \cdot x+b_{1} \\
& y(w, k)=a_{2} \cdot x+b_{2}
\end{aligned}
$$

The values of the two coordinates are (rel. 18):

$$
\begin{aligned}
& x(u, v, w, k)=\frac{b_{1}(u, v)-b_{2}(w, k)}{a_{2}(w, k)-a_{1}(u, v)} \\
& y(u, v, w, k)=b_{1}(u, v)+a_{1}(u, v) \frac{b_{1}(u, v)-b_{2}(w, k)}{a_{2}(w, k)-a_{1}(u, v)}
\end{aligned}
$$

Starting from the two coordinates, which signify the mapping (correspondence) with the values of the pixel in the original image, the pixel in the final image will be defined by the relation:

$$
I^{\prime}(x, y)=I[x(u, v, w, k), y(u, v, w, k)]
$$

Although the parameters $u ; v ; w ; k$ have values in the interval $[0,1]$, this interval can be extended to interpolate on the whole distance of the initial image.

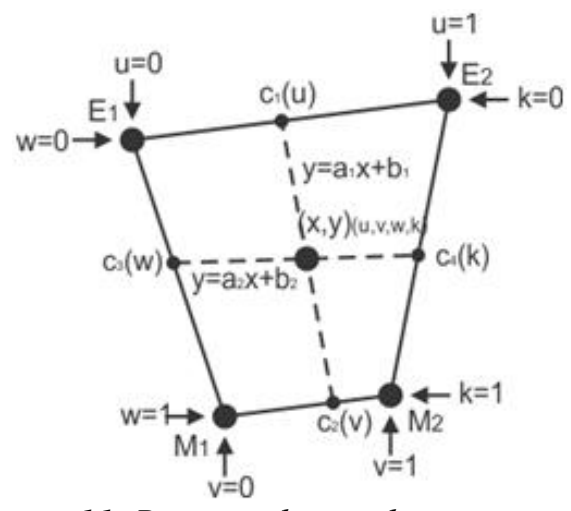

Figure 11. Rectangular grid in perspective distortion correction

The corrected image can be used by a facial recognition algorithm based on the model. When implementing the facial recognition algorithm, we use only the area corresponding to the face, and the interest area is obtained by cutting from the corrected image an area corresponding to the human face (rel. 20):

$x_{\min }=x_{E}-D_{M E}$
$x_{\max }=x_{M}+D_{M E}$
$y_{\min }=y_{L E}-D_{M E}$
$y_{\max }=y_{R E}-D_{M E}$

where $x_{E}$ is the $x$ coordinate of the line of the eyes, $x_{M}$ is the $x$ coordinate of the mouth, $y_{L E}$ is the $y$ coordinate of the left eye, $y_{R E}$ is the $y$ coordinate of the right eye, and $D_{M E}$ is the distance between the mouth and the middle of the line of the eyes.

The results obtained by applying the correction of the eyes' detection, as well as by applying geometric corrections, allow the obtaining of some classification rates superior to the case when the processing is missing. By applying corrections, one can obtain rates of $72.67 \%$, and $70.12 \%$, respectively, compared to values of $63.12 \%$ and $60.03 \%$ respectively, for the case when these corrections were not implemented.

In the case of the classification with neural networks, the error rates that were obtained for the two resolutions of the images (converted as input vectors) are higher if the corrections were applied. Values of $76.44 \%$ and $74.12 \%$ are obtained, respectively, as compared to values of $64.43 \%$ and $62.18 \%$ respectively. We should mention the fact that recognition problems occur, on the one hand, due to 
errors of rotation corrections or where proper detection of the eyes could not be performed because of the degraded image, and, on the other hand, because of the selections of the images from the database.

\section{Conclusions}

From the observations made during the analysis, the perspective correction is useful only if the grid in the original image is correctly detected. Otherwise, the introduced deformities can be major for a small deviation of the points of the extremities of the mouth.

It is estimated that superior results can be obtained by means of a nonlinear interpolation grid that would consider other facial features such as the nose or the limits of the face area. Particular attention will be paid to the detection of the contour of the mouth, in order to use this information as the upper lip contour does not radically change its form when the facial expression changes.

\section{References}

[1] X. Lu, "Image analysis for face recognition", Personal notes, Dept. of Computer Science \& Engineering, Michigan State University, East Lansing, MI, May 2003

[2] W. Zhao, R. Chellappa, P.J. Philips, A. Rosenfeld, "Face recognition: a literature survey", ACM Computing Surveys, vol. 35, no. 4, p. 399-458, Dec. 2003

[3] R. Jafri, H.R. Arabnia, "A survey of face recognition techniques", Journal of Information Processing Systems, vol. 5, no. 2, p. 41-68, 2009

[4] M. Turk, A. Pentland, "Eigenfaces for recognition", Journal of Cognitive Neuroscience, Vol. 3, no. 1, pp. 71-86, 1991

[5] D.L. Swets, J.J. Weng, "Using discriminant eigenfeatures for image retrival", IEEE Transactions on Pattern Analysis and Machine Intelligence, vol. 18, 1996

[6] A.M. Martinez, AC. Kak, "PCA versus LDA", IEEE Transactions on Pattern Analysis and Machine Intelligence, vol. 23, no. 2, 2001.

[7] M.S. Bartlett, H.M. Lades, T.J. Sejnowski, "Independent component representation for face recognition", Proceedings of SPIE, 1998

[8] C. Beumier, M. Acheroy, "Automatic face recognition", Proceedings symposium IMAGING. Eindhoven, The Netherlands, pp. 77-89, 2000

[9] S. Roy, S. Podder, "Face detection and its applications", International Journal of Research in Engineering \& Advanced Technology, Volume 1, Issue 2, April-May, 2013

[10]R.-L. Hsu, M. Abdel-Mottaleb, A.K. Jain, "Face detection in color images", IEEE Transactions on Pattern Analysis and Machine Intelligence, vol. 24, no. 5, pp. 696-706, May 2002

[11]G. Loy, A. Zelinsky, "Fast radial symmetry for detecting points of interest", IEEE Transactions on Pattern Analysis and Machine Intelligence, vol. 25, no. 8, pp. 959-973, Aug 2003.

[12]R. Oancea, C. Molder, "Locating facial features in images with uncontrolled context", IEEE International Symposium on IT in Medicine \& Education (ITME 2011), Guangzhou, China, 2011

[13]A. Abiol, L. Torres, E.J. Delp, "Optimum color space for skin detection", ICIP, 2001

[14]M.J. Jones, J.M. Rehg, "Statistical color models with application to skin detection", Int. Journal of Computer Vision, Vol. 46, No. 1, pp. 81-96, 2002

[15]C. Prema, D. Manimegalai, "Survey on skin tone detection using color space", International Journal of Applied Information Systems (IJAIS), vol. 2, no. 2, 2012

[16]B.D. Zarit, B.J. Super, F.K.H. Quek, "Comparison of five color models in skin pixel classification", in Recognition, Analysis and tracking of faces and Gesture in Real-Time System, p. 58-63, 1999 
[17]A.S.M. Sahail, P. Bhattacharya, "Detection of facial feature points using anthropometric face model", Signal Processing for Image Enhancement n Multimedia Processing, vol. 34, Springer, pp 189-200, 2007

[18]J.P. Batista, "Locating facial feature using an anthropometric face model for determining the gaze of face in image sequences", ICIAR, vol. 4633, pp. 839-853, 2007

[19]E. Hjelmås, B.K. Low, "Face detection: a survey", Computer Vision and Image Understanding, vol. 83, pp. 236-274, 2001.

[20]C. Lin, K.-C. Fan, "Human face detection using geometric triangle relationship", Proceedings of the 16th International Conference on Pattern Recognition (ICPR '00), vol. 2 , Barcelona, Spain, pp. 941-944, Sep. 2000

[21]C. Lin, K.-C. Fan, "Triangle-based approach to the detection of human face", Pattern Recognition, vol. 34, no. 6, pp. 1271-1284, 2001.

[22] S.Nedevschi, RI. Peter, A. Mandrut, "PCA type algorithm applied in Face Recognition", Proceedings of 2012 IEEE Intelligent Computer Communication and Processing, ClujNapoca, pp. 2167-171, August 30-September 1, 2012.

[23] Molder Cristian, Oancea Romana, "Appearance-based Facial Detection for Recognition", 9th International Conference on Communications (COMM), 2012

[24]J-K. Kourosh, S.-Z. Hamid, "Radon transform orientation estimation for rotation invariant texture analysis", in IEEE Transaction on Pattern Analysis and Machine Intelligence, vol. 27, no. 6, pp. 1004-1008, June 2005

[25]S. M. Murtoza Habib, Nawsher Ahamed Noor, Mumit Khan, "Skew angle detection of Bangla script using Radon transform ", 9th International Conference on Computer \& Information Technology, pp. 104-109, Bangladesh, 2006, http://www.academia.edu/638986/Skew_angle_detection_of_bangla_script_using_radon _transform 